\title{
Modelling Synergy using Manifest Categorical Variables
}

\author{
A lexander von E ye \\ Michigan State University, USA \\ Christof Schuster \\ University of Michigan, USA \\ William M. Rogers \\ Michigan State University, USA
}

\begin{abstract}
This paper discusses methods to model the concept of synergy at the level of manifest categorical variables. First, a classification of concepts of synergy is presented. Additive and nonadditive concepts of synergy are distinguished. Most prominent among the nonadditive concepts is superadditive synergy. Examples are given from the natural sciences and the social sciences. Modelling focuses on the relationship between the agents involved in a synergetic process. These relationships are expressed in form of contrasts, expressed in effect coding vectors in design matrices for nonstandard loglinear models. A method by Schuster is used to transform design matrices such that parameters reflect the proposed relationships. An example reanalyses data presented by Bishop, Fienberg, and Holland (1975) that describe the development of thromboembolisms in women who differ in their patterns of contraceptive use and smoking. A lternative methods of analysis are compared. Implications for developmental research are discussed.
\end{abstract}

The concept of "synergy" is important in a wide variety of scientific contexts. The term generally applies to situations involving the action of two or more agents in effecting an outcome. The relationship between the agents, and their individual relationships with the outcome, in part allow the definition of different types of synergy. It is important to make a

Requests for reprints should be sent to Professor A lexander von Eye, Michigan State University, Department of Psychology, 119 Snyder Hall, MI 48824-1117, USA.

The authors are indebted to Maria Wong and Robert A. Zucker for commenting on earlier versions of this paper. A lexander von Eye's work on this paper was supported in part by NIAA A grant No. ZR01 A A 07065.

(c) 1998 The International Society for the Study of Behavioural Development 
distinction between numerical or statistical definitions of synergy, and definitions based on empirical mechanisms. It is likely that a wide variety of agent-outcome relationships can be classified in a general synergy taxonomy for the purposes of analysis and statistical conclusion. One of the purposes of this paper is to present such a statistical method, using manifest categorical variables, which would be usable in a variety of contexts. It is quite another issue to examine the empirical mechanisms of synergy, as they are germane to the particular field in question. For example, the empirical relationship among psychotherapies in their effects on mental illness is surely not the same type of empirical relationship as exists among chemical insecticides in their effects on crop pests. Both are nevertheless able to be analysed using the same methodology and perhaps the same statistical model.

\section{SYNERGY: DEFINITION AND USE OF TERM}

Classifying synergy relationships based on numerical criteria essentially delineates the relationship between quantities associated with the agents and the quantity associated with an outcome. This numerical relationship, at least from the perspective of statistical modelling, may fall into two broad classifications: additive and nonadditive. Additive synergy refers to a situation in which the total effect is simply the sum of the independent effects for each agent. In this situation, there need not be relationships between the agents, but only a similar relationship between each agent and the desired effect (e.g. team members' contributions to total effort in ropepulling contest). A nother case, which might be considered a subset of additive synergy, is conditionally additive synergy. This is a situation where the action of the entire set of agents is required to produce a desired effect. For any agent to transfer an effect, the actions of other agents are necessary. Any agent acting in isolation cannot produce the effect.

The distinction between additive and conditionally additive conceptualisations of synergy can become hazy when the quantitative nature of the outcome is in question. For instance, if one is examining a quantitative outcome variable with a critical threshold level at which a qualitative change takes place, simple additive effects can appear conditionally additive. In a situation where the true relationship is additive, two individual agents' effects may fall below threshold individually, suggesting no qualitative effect, whereas their sum exceeds threshold, producing what appears to be an effect conditional on both agents' activity.

Finally, the relationship can be seen as nonadditive, which includes the subclassifications of superadditive, subadditive, and isolated synergy. Superadditive synergy occurs when the "whole is greater than the sum of parts", and subadditive synergy is denoted by the "whole being less than 
the sum of parts". Numerically, these relationships are defined by both an additive component for each agent's effect on the outcome, and an interactive (or multiplicative) component for the relationships among agents in affecting the outcome. Isolated synergy involves the interactive component among agents, but without the influence of any additive effects for individual agents. Hybrid models, which contain both interactive terms and a subset of additive effects for each agent, are also conceivable.

The types of synergy can be distinguished by type and degrees of restrictions the statistical models place on the relationships between agents and effects. A lthough all types implicitly posit that effects caused by agents can or do exist, they differ in how the relationships between agents are conceptualised, and how the concepts of individual effects differ from the composite effect. It should be noted that synergy has only thus far been defined numerically, by magnitude of effects on an outcome. The present paper proposes also modelling the relationships between agents.

A linkage of conceptual and empirical notions of synergy can be gained by examining scientific domains in which the term "synergy" has been invoked and used to explain empirical results. The superadditive notion of synergy is common across many domains of scientific literature, and is often considered the "classical" conceptual type of synergy. Examples can be found in both the natural sciences and social sciences. The following paragraphs first give a selection of examples of the use of the synergy concept in the natural sciences. Examples from the social sciences follow.

Synergy in the Natural Sciences: Examples of Use. Bennett, Mlady, Fleshner, and Rose (1996) found that two chemical treatments which did not cause behavioural impairment when administered separately, did cause marked behavioural impairment when co-administered. Arnold et al. (1996) studied the effects of environmental oestrogenic compounds, and found that when administered in concert, oestrogenic compounds increased their individual potencies to over one thousand times their original individual potency. D rasner (1988), in studying the antinociceptive effects of morphine and clonidine, found that either in isolation had no effect, whereas their combination showed antinociceptive effects. Seale et al. (1987) demonstrated that caffeine and other subconvulsant compounds, when combined created convulsive effects in rats.

In epidemiology, Worcester (1971; see Bishop et al., 1975) asked whether thromboembolisms are more frequent in individuals who both smoke and take oral contraceptives than in individuals who either only smoke or only take oral contraceptives, or neither. The two agents in this example are smoking and the oral contraceptive. Bishop et al. (1975) analysed this data set using the first, less restrictive of the foregoing two 
definitions. Results suggested that the two agents indeed can be seen as synergists. (Later sections of this paper use these data again.)

In biochemistry, Morse, McK inlay, and Spurr (cited from McCullagh \& Nelder, 1983) asked whether the insecticide, carbofuran, and the synergist, piperonyl butoxide, are jointly more efficient than either in controlling the grasshopper Melanoplus sanguinipes.

Synergy in the Social Sciences: Examples of Use. The applications of the superadditive concept are not limited to the natural sciences. Ketter (1992) found that symptoms associated with bipolar disorder were refractory to either of two psychoactive medications, but responsive to the combination. Gunzburg (1995) applied individual, group, and marital therapy methods, and demonstrated the synergistic functioning of the three in combination.

Wilens, Spencer, Biederman, and Wozniak (1995) raised the issue of synergy of medical drugs used together for treatment of psychiatric disorders in children and adolescents. The authors detail guidelines that can be used for determining the appropriateness of using multiple agents (drugs) in pharmacotherapy. In a comparable context, Buckley and Schulz (1996) discussed the potential for synergy between novel antipsychotic drugs and modern psychosocial therapies in the treatment of schizophrenia and affective disorders.

In a psychotherapeutic context, Lodeon (1986) attempted to establish synergy between the desired healing behaviour and the symptom presented at consultation. The reported symptom was in two cases that a young male and a young female were unable to consummate their respective marriages.

In an effort to specify a mathematical model of olfactory perception, Laffort, Etcheto, Patte, and Marfaing (1989) combined the experimental effectiveness of mixtures with the power law exponent of the components. The authors define a coefficient that can be used to derive curves of olfactory iso-intensity. From these curves the authors conclude that the coefficient has "integrative strength" that allows one to depict synergy of odour concentrations.

A lso in psychophysiology, Ayya and Lawless (1992) compared experimentally the sweetness of single sweeteners and 50:50 mixtures of sweeteners. Results suggested that sweeteners act synergistically. In addition, based on comparisons of frequencies, the authors interpreted their results as supporting the concept of superadditivity.

At a more theoretical level, Nelson (1996) discussed philosophical and psychologial approaches to consciousness. The author proposes that the research on metacognition can produce synergy between the two 
approaches by specifying constraints on the range of acceptable theories and by guiding development of new theories.

In biology, synergy is often used to describe the coordination of two muscles, for instance, of flexors and extensors. Aruin, A lmeida, and Latasch (1996) analysed the behaviour of Down syndrome patients who displayed lack of synergy of these muscles in elbow or wrist movements. The authors observed that the patients show simultaneous bursts of both flexor and exensor, and discuss theories explaining the patients' adaptation to problems that can result from this behaviour.

Houston and Doan (1996) investigated the synergy of negative and positive political advertising campaigns. The authors state that both types of information in campaigns act in synergy and direct the voters' attention to either the bad or the good features of the competing candidates.

Most prominent in developmental research is Gottlieb's (1992) concept of synergy. The author proposes that the "cause of development-what makes development happen-is the relationship of the two components, not the components themselves" (pp. 161-162). Gottlieb's term component is used in a fashion parallel to the present term agent. Sample components/ agents are organisms, environment, or the sensory system.

Though not as prevalent in published research, subadditive effects of agent combinations have also been demonstrated. Broglia and Brunori (1994) investigated the effects of high sucrose concentration and low temperature on maize pollen viability. It was found that low temperature or high sucrose concentration presented in isolation caused maize pollen to lose viability quickly. However, both presented simultaneously had no such effect. Miaskowski (1993) examined the effects of spinal chemical concentrations on nociceptive thresholds in male rats. It was found that supraspinal DDPE and spinal DAMGO both altered nociceptive thresholds in isolation, but their concurrent administration showed no synergistic effects. It should be noted that the quantitative nature of outcome variables in these examples is sufficiently vague as to suggest the classification of synergy into subadditive versus superadditive categories may be arbitrary. Without a proper understanding of the mechanisms of the relationship, the subtractive or additive effects of agent combinations cannot be determined. It can, however, be shown that the relationship is something more than simple additive synergy.

The empirical mechanisms behind synergistic effects in the biological and biochemical sciences are varied, but two are notable-chemical interaction between the agents, and interactive relationship in the biological system. An example of the first concept is the simple chemical reaction. The presence of multiple chemical agents may transform any given agent into a slightly different chemical compound, having different effects. The latter type is likely more predominant, the prototypical 
example being two chemical agents competing for the same receptor sites in a neurochemical system. The effectiveness of psychoactive drugs is predicated on the fact that they block receptor sites from natural neurotransmitters associated with behavioural disorders.

The concept of synergy is related to the causal Wedge (see Sobe1, 1995; von Eye \& Brandtstädter, in press). The Wedge conceptualises a causal relationship where one or more causes lead to the same effect. The concept of the Weak Wedge is open to the interpretation that a goal can be reached through more than one cause. Each of the causes is sufficient yet not necessary for the effect to be observed. Synergy can be viewed as a special case of the wedge in the sense that two or more agents are always both sufficient and necessary for an effect (see Fig. 1).

The present article is concerned with modelling synergy at the level of manifest categorical variables. We propose log-linear models for modelling the concept of synergy. Focus is on manifest categorical variables. Based on a method proposed by Schuster (in preparation), design matrices are specified that lead to parameters that reflect the contrasts under study. The methods proposed here: (1) operate at the level of manifest variables; (2) use categorical variables; and (3) define relationships among variables at the level of categories of variables rather than variables as such.

\section{MODELLING SYNERGY USING NONSTANDARD LOG-LINEAR MODELS}

The present modelling approach has two goals. First, we specify models that describe the data in a satisfactory fashion. Second, we attempt

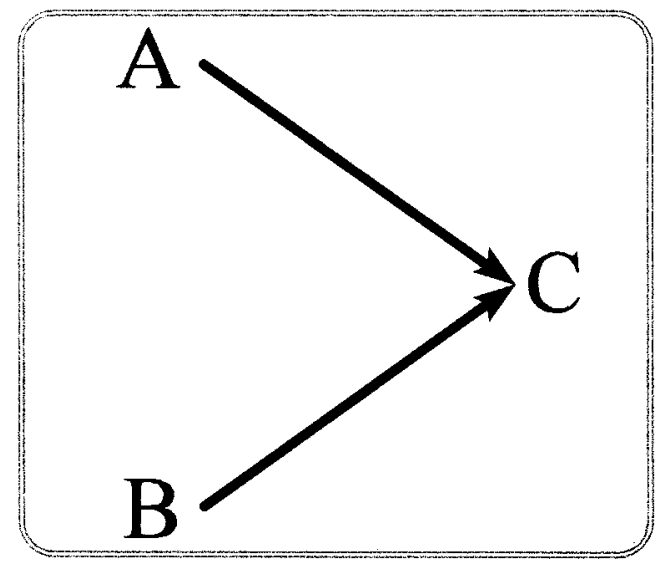

FIG. 1 Synergetic activity of two agents. 
parameter interpretation. Although in many instances, researchers are satisfied with fitting models, and parameters are of lesser importance (see Sloane \& Morgan, 1996), the present approach requires parameter interpretation. It is not sufficient to provide a model that fits. One has to show in addition that the contrasts specified to test a particular hypothesis capture significant portions of the variability in a table. This section first describes the long-linear models used for modelling. Subsequently, problems of parameter interpretation will be addressed.

\section{Standard and Nonstandard Log-Linear Models}

Consider the following log-linear model

$$
\mu=\mathrm{X} \lambda \text {, }
$$

where $\mu=\log \mathrm{F}, \mathrm{F}$ is the array of expected cell frequencies, $\mathrm{X}$ is the indicator (design) matrix, and $\lambda$ is the parameter vector. Standard, hierarchical log-linear models of the form

$$
\mu=\lambda_{0}+\mathrm{X}_{m} \lambda_{m}+\mathrm{X}_{i} \lambda_{i},
$$

where $\lambda_{0}$ is the parameter for the constant, subscript $m$ indexes main effects, and $i$ indexes interactions, are often specified hierarchically, such that higher-order terms imply the existence of lower order terms. For example, the interaction, $[A, B]$, implies that all main effect terms for variable $A$ and all main effect terms for variable $B$ are part of the model.

Nonstandard log-linear models (R indskopf, 1990; von E ye, Kreppner, \& Weßels, 1994; von Eye \& Spie1, 1996) can be given as

$$
\mu=\lambda_{0}+\mathrm{X}_{m} \lambda_{m}+\mathrm{X}_{i} \lambda_{i}+\mathrm{X}_{s} \lambda_{s}
$$

where $s$ indexes the "special vectors" (Clogg, E liason, \& G rego, 1990) that cannot be derived from standard main effect and interactions. These special vectors express contrasts that can be compared to contrasts in analysis of variance post-hoc tests.

When modelling such concepts as synergy we use the elements in equation 3 as follows. The first three terms on the right-hand side of the equation (i.e. $\lambda_{0}, \mathrm{X}_{m} \lambda_{m}$, and $\mathrm{X}_{i} \lambda_{i}$ ) express those relationships among variables that are not specific to the concept under study. These three terms form what we will call the base model. Hypothesised deviations from the base model are parameterised in the fourth term (i.e. $\mathrm{X}_{s} \lambda_{s}$ ). This term subsumes the vectors that are needed to model the concept under study. This term will be called the specific part of the model. 


\section{Parameter Interpretation in Nonstandard Log- Linear Models}

Parameter interpretation in log-linear models has been an issue of long debates. Whereas some contributions focused on the meaning of parameters, for instance, the meaning of main effect parameters in the presence of interaction parameters (e.g. Alba, 1988; Holt, 1979; Long, 1984), others discussed techniques for interpretation of parameters (e.g. Elliott, 1988). The reason for these discussions is obvious: parameters do not necessarily reflect the hypotheses specified in the design matrix, $X$.

One of the problems encountered when interpreting parameters can be described as follows. The relationship between the design matrix, $\mathrm{X}$, and the parameters is

$$
\lambda=\left(\mathrm{X}^{\prime} \mathrm{X}\right)^{-1} \mathrm{X}^{\prime} \mu,
$$

where both, the base model and the contrasts of interests, are included in $\mathrm{X}{ }^{1}$ This relationship reflects the contrasts of interest only if $\mathrm{X}$ is orthogonal. As soon as $\mathrm{X}$ is no longer orthogonal, interpretation of parameters becomes complicated if not impossible. Consider the following two examples.

Example 1: Parameters from Saturated $2 \times 2 \times 2$ Table. In a $2 \times 2 \times 2$ table a saturated log-linear model is specified. The design matrix for th model appears in Table 1. Applying equation 4 one obtains the parameter interpretation given in Table 2.

TABLE 1

Design Matrix for Saturated Log-Linear Model in $2 \times 2 \times 2$ Table

\begin{tabular}{|c|c|c|c|c|c|c|c|c|}
\hline \multirow{3}{*}{$\begin{array}{c}\text { Cell } \\
\text { Indices } \\
111\end{array}$} & \multicolumn{8}{|c|}{ Effect Coding Vectors } \\
\hline & \multirow{2}{*}{$\frac{\text { Constant }}{1}$} & \multicolumn{3}{|c|}{ Main Effects } & \multicolumn{4}{|c|}{ Interactions } \\
\hline & & 1 & 1 & 1 & 1 & 1 & 1 & 1 \\
\hline 112 & 1 & 1 & 1 & -1 & 1 & -1 & -1 & -1 \\
\hline 121 & 1 & 1 & -1 & 1 & -1 & 1 & -1 & -1 \\
\hline 122 & 1 & 1 & -1 & -1 & -1 & -1 & 1 & 1 \\
\hline 211 & 1 & -1 & 1 & 1 & -1 & -1 & 1 & -1 \\
\hline 212 & 1 & -1 & 1 & -1 & -1 & 1 & -1 & 1 \\
\hline 221 & 1 & -1 & -1 & 1 & 1 & -1 & -1 & 1 \\
\hline 222 & 1 & -1 & -1 & -1 & 1 & 1 & 1 & -1 \\
\hline
\end{tabular}

${ }^{1}$ Note that equation 4 describes the relationship between $\mathrm{X}$ and the parameters. Estimation is still maximum likelihood. 
TABLE 2

Parameters in Saturated Model for $2 \times 2 \times 2$ Table

\begin{tabular}{cc}
\hline Parameter & Estimate \\
\hline$\lambda_{0}$ & $1=8\left(\mu_{1}+\mu_{2}+\mu_{3}+\mu_{4}+\mu_{5}+\mu_{6}+\mu_{7}+\mu_{8}\right)$ \\
$\lambda_{A}$ & $1=8\left(\mu_{1}+\mu_{2}+\mu_{3}+\mu_{4}-\mu_{5}-\mu_{6}-\mu_{7}-\mu_{8}\right)$ \\
$\lambda_{B}$ & $1=8\left(\mu_{1}+\mu_{2}-\mu_{3}-\mu_{4}+\mu_{5}+\mu_{6}-\mu_{7}-\mu_{8}\right)$ \\
$\lambda_{C}$ & $1=8\left(\mu_{1}-\mu_{2}+\mu_{3}-\mu_{4}+\mu_{5}-\mu_{6}+\mu_{7}-\mu_{8}\right)$ \\
$\lambda_{A B}$ & $1=8\left(\mu_{1}+\mu_{2}-\mu_{3}-\mu_{4}-\mu_{5}-\mu_{6}+\mu_{7}+\mu_{8}\right)$ \\
$\lambda_{A C}$ & $1=8\left(\mu_{1}-\mu_{2}+\mu_{3}-\mu_{4}-\mu_{5}+\mu_{6}-\mu_{7}+\mu_{8}\right)$ \\
$\lambda_{B C}$ & $1=8\left(\mu_{1}-\mu_{2}-\mu_{3}+\mu_{4}+\mu_{5}-\mu_{6}-\mu_{7}+\mu_{8}\right)$ \\
$\lambda_{A B C}$ & $1=8\left(\mu_{1}-\mu_{2}-\mu_{3}+\mu_{4}-\mu_{5}+\mu_{6}+\mu_{7}-\mu_{8}\right)$ \\
\hline
\end{tabular}

A comparison of the estimates in Table 2 with the contrasts in Table 1 show that the estimates perfectly reflect the specified effects.

Example 2: Parameters from Nonorthogonal Design Matrix. Table 3 displays the design matrix for a $4 \times 4$ cross-tabulation. Only the main effects are considered for the base model. For the specific part of the model two additional vectors are introduced.

In contrast to the parameter estimates in the first example, the present estimates do not reflect the contrasts specified in Table 3. This can be

TABLE 3

Design Matrix for Non-standard Log-Linear Model for $4 \times 4$ Cross-tabulation

\begin{tabular}{cccccccccc}
\hline $\begin{array}{c}\text { Cell } \\
\text { Index }\end{array}$ & Constant & \multicolumn{1}{c}{ Main Effects Rows } & Main Effect Columns & \multicolumn{2}{c}{ Contrasts } \\
\hline 11 & 1 & 1 & 0 & 0 & 1 & 0 & 0 & 0 & 0 \\
12 & 1 & 1 & 0 & 0 & 0 & 1 & 0 & 0 & 0 \\
13 & 1 & 1 & 0 & 0 & 0 & 0 & 1 & 0 & 0 \\
14 & 1 & 1 & 0 & 0 & -1 & -1 & -1 & -1 & 0 \\
21 & 1 & 0 & 1 & 0 & 1 & 0 & 0 & 0 & 0 \\
22 & 1 & 0 & 1 & 0 & 0 & 1 & 0 & 0 & 0 \\
23 & 1 & 0 & 1 & 0 & 0 & 0 & 1 & 0 & 0 \\
24 & 1 & 0 & 1 & 0 & -1 & -1 & -1 & -1 & 0 \\
31 & 1 & 0 & 0 & 1 & 1 & 0 & 0 & 0 & 0 \\
32 & 1 & 0 & 0 & 1 & 0 & 1 & 0 & 0 & 0 \\
33 & 1 & 0 & 0 & 1 & 0 & 0 & 1 & 0 & 0 \\
34 & 1 & 0 & 0 & 1 & -1 & -1 & -1 & 1 & 0 \\
41 & 1 & -1 & -1 & -1 & 1 & 0 & 0 & 0 & -1 \\
42 & 1 & -1 & -1 & -1 & 0 & 1 & 0 & 0 & -1 \\
43 & 1 & -1 & -1 & -1 & 0 & 0 & 1 & 0 & 1 \\
44 & 1 & -1 & -1 & -1 & -1 & -1 & -1 & 1 & 1 \\
\hline
\end{tabular}


illustrated using the parameter estimate for the first of the special vectors. This estimate is given by

$$
\begin{aligned}
\lambda_{x 1}= & \frac{\mu_{11}}{16}+\frac{\mu_{12}}{16}+\frac{\mu_{13}}{8}-\frac{\mu_{14}}{4} \\
& +\frac{\mu_{21}}{16}+\frac{\mu_{22}}{16}+\frac{\mu_{23}}{8}-\frac{\mu_{24}}{4} \\
& -\frac{\mu_{31}}{8}-\frac{\mu_{32}}{8}-\frac{\mu_{33}}{16}+\frac{5 * \mu_{34}}{16} \\
& +0 * \mu_{41}+0 * \mu_{42}-\frac{3 * \mu_{43}}{16}+\frac{3 \mu_{44}}{16} .
\end{aligned}
$$

This linear function is virtually uninterpretable and unrelated to the original hypothesis, given in the second last column in Table 3 . To obtain parameters that do reflect the contrasts specified in the original design matrix, $X$, Schuster (in preparation) proposed the following transformation. Consider matrix $H$ where $H^{\prime}$ has the same dimensions as $\mathrm{X}$ and:

$\mathrm{H} \mu$ specifies the contrasts of interest

$$
C(\mathrm{X})=C\left(\mathrm{H}^{\prime}\right) \quad[C(\mathrm{~A})=\text { column space of } \mathrm{A}]
$$

Let

$$
\mathrm{X}^{*}=\mathrm{H}^{\prime}(\mathrm{HH})^{-1},
$$

and substitute $\mathrm{X}^{*}$ for $\mathrm{X}$ when estimating $\lambda$. This leads to

$$
\begin{aligned}
\lambda & =\left[\left(\mathrm{X}^{*}\right)^{\prime} \mathrm{X}^{*}\right]^{-1}\left(\mathrm{X}^{*}\right)^{\prime} \mu \\
& =\left[(\mathrm{HH})^{-1} \mathrm{HH}^{\prime}(\mathrm{HH})^{-1}\right]^{-1}(\mathrm{HH})^{-1} \mathrm{H} \mu \\
& =H \mu .
\end{aligned}
$$

Equation 7 shows that the parameters are related to the contrasts as specified in the original design matrix when the estimation uses $\mathrm{X}^{*}$ instead of the original design matrix, $X$. Thus, the researcher only has to perform the following four steps to obtain parameter estimates that can be interpreted as specified in the original matrix, $X$ : (1) set up $X$; (2) determine $X^{\prime}=H$; (3) calculate $X^{*}$; and (4) perform analysis using $X^{*}$ instead of $X$.

Performing these four steps yields results with the following characteristics. First, the overall model fit is identical to model fit from $\mathrm{X}$ because $C(\mathrm{X})=C\left(\mathrm{X}^{*}\right)=C(\mathrm{H})$. Second, the hypotheses specified in original design matrix, $X$, are reflected in parameters. Third, parameters, standard errors, parameter intercorrelations, etc. are part of standard output of readily available software (e.g. S+, CDAS), and fourth, all statistical results are obtained simultaneously. 
We will illustrate that the transformation described in equations 6 and 7 yields interpretable parameters in the data example in the section on four synergy models. In the following section we show how to model synergy.

\section{MODELLING SYNERGY}

A s was indicated in the first section of this paper, synergy requires two or more agents to co-operate toward a common goal. In the following sections we distinguish the following three variants of synergy:

1. Independent Agents' Synergy. Each of two or more agents displays the same effect on the dependent measure. To explain the synergy effect, at least two agents must have significant effects. For an example consider a student who is talented and invests effort in her homework. Under the Independent Agents' Synergy hypothesis both the student's talent and her effort account for a significant portion of success in homework. However, there is no additional effect when both talent and effort are taken into account. This concept can be viewed parallel to the first definition of synergy given in the introduction.

2. Joint Agents' Synergy. Each of two or more agents displays the same effect on the dependent measure. Two types of effect must exist to explain the synergy effect: (1) the effects of two or more agents; and (2) special interactions between the agents. If theory requires the interaction(s) to exist, there is superadditivity in the sense given in the second definition in the introduction. If there are two agents, an interaction must exist between these two. If there are more than two agents, theory may require that either all interactions must exist for complete Joint Agents' Synergy, or only the highest-order interaction.

3. Isolated Synergy. Here, theory requires only the interaction to exist. There is no need for single agents' effects to exist. When there are more than two agents, theory may require that all interactions or just the highest-order interaction must exist (see equation 2).

Hybrid forms of synergy can also be considered. For example, a hybrid between the second and the third variant would be a case where, in addition to interactions, only some of the agents display effects on the dependent measure. To illustrate these three concepts of synergy we present in the next section a data example.

\section{Synergy in the Development of} Thromboembolisms: A Data Example

Bishop et al. (1975) analysed data presented by Worcester (1971) on the causation of thromboembolisms. The three variables, Use of Oral 
Contraceptives $(U$, yes $=1$, no $=2)$, Smoker $(S$, yes $=1$, no $=2)$, and Presence of Thromboembolisms $(T$, yes $=1$, no $=2)$ were observed in a sample of $N=174$ respondents. The research question was whether U se of Oral Contraceptives and Smoking can be viewed as synergists for the development of thromboembolisms. Table 4 displays the observed frequencies of the cross-tabulation of $T, S$, and $U$, along with the expected cell frequencies, estimated under the base model, $[S, U][T]$, and the residuals.

\section{Four Synergy Models}

Here, we will review some of the results presented by Bishop et al. (1975, pp. 112-114) and reanalyse these data using the methods proposed in this paper. Specifically, we discuss the following five models: (1) the base model, $[U, S][T]$, of no relationships between predictors and criterion; (2) the nonhierarchical model log-frequency proposed by Bishop et al. (1975),

$$
\mu=\lambda_{0}+\lambda_{1}^{U}+\lambda_{2}^{S}+\lambda_{3}^{T}+\lambda_{12}^{U S}+\lambda_{123}^{U S T},
$$

(3) the Independent A gents' Synergy Model where the two agents, $U$ and $S$, simultaneously have effects on $T$, but there is no need to accommodate surplus variability which would indicate superadditivity; (4) the Joint A gents' Synergy Model which enriches the Independent Agents' Synergy Model by including a term that accounts for the interaction that results in superadditivity; and (5) the Isolated Synergy Model which only considers the agents' interactions. Model 2 was reported in Bishop et al. (1975).

The Independent Agents' Synergy Model. This only requires all agents to have the desired effect. These effects manifest in the same category or categories. No interaction is required. The agents whose effects are significant operate in synergy. The design matrix for this model appears in Table 5 .

TABLE 4

Cross-tabulation of $T, S$, and $U$

\begin{tabular}{cccccc}
\hline TSU & Observed & Expected & Residual & Std. Resid. & Adj. Res. \\
\hline 111 & 14.00 & 4.34 & 9.65517 & 4.63206 & 5.546 \\
112 & 7.00 & 16.66 & -9.65517 & -2.36584 & -5.546 \\
121 & 12.00 & 7.66 & 4.34483 & 1.57035 & 1.987 \\
122 & 25.00 & 29.34 & -4.34483 & -0.80206 & -1.987 \\
211 & 2.00 & 4.97 & -2.96552 & -1.33082 & -1.609 \\
212 & 22.00 & 19.03 & 2.96552 & 0.67972 & 1.609 \\
221 & 8.00 & 19.03 & -11.03448 & -2.52919 & -4.136 \\
222 & 84.00 & 72.97 & 11.03448 & 1.29179 & 4.136 \\
\hline
\end{tabular}


TABLE 5

Design Matrix for Independent Agents' Synergy Model for Thromboembolism Data

\begin{tabular}{rrrrrrrrr}
\hline$T S U$ & $F$ & $c$ & $T$ & $S$ & $U$ & $x_{1}$ & $x_{2}$ & $S \times U$ \\
\hline 111 & 14 & 1 & -1 & -1 & -1 & 1 & 1 & 1 \\
112 & 7 & 1 & -1 & -1 & 1 & 1 & 0 & -1 \\
121 & 12 & 1 & -1 & 1 & -1 & 0 & 1 & -1 \\
122 & 25 & 1 & -1 & 1 & 1 & 0 & 0 & 1 \\
211 & 2 & 1 & 1 & -1 & -1 & -1 & -1 & 1 \\
212 & 22 & 1 & 1 & -1 & 1 & -1 & 0 & -1 \\
221 & 8 & 1 & 1 & 1 & -1 & 0 & -1 & -1 \\
222 & 84 & 1 & 1 & 1 & 1 & 0 & 0 & 1 \\
\hline
\end{tabular}

The contrasts specific to the Independent Agents' Synergy Model are expressed in Vectors, $x_{1}$ and $x_{2}$. Vector $x_{1}$ contrasts cells 111 and 112 on the one hand side with cells 211 and 212 on the other. This contrast reflects the hypothesis that smokers suffer from thromboembolisms at increased rates, and that regardless of whether they do or do not take oral contraceptives. The second specific vector, $x_{2}$, contrasts cells 111 and 121 with 211 and 221, reflecting the hypothesis that users of oral contraceptives suffer from thromboembolisms at increased rates, and that regardless of whether they smoke or not. Results of this analysis will be interpreted in terms of a synergetic effect if (1) the model fits and (2) both of the specific vectors, $x_{1}$ and $x_{2}$, are significant.

The Joint Agents'Synergy Model. This proposes that it is not sufficient if two or more agents work toward the same goal. R ather, there must be an effect beyond what the independent agents can achieve. This "effect beyond" has been called superadditivity. In the present context we specify an additional vector to express superadditivity. This vector reflects the cooperation between the two agents. Here, we hypothesise that individuals who both smoke and take oral contraceptives suffer from increased rates of thromboembolisms, beyond what can be explained by the effects of smoking and taking oral contraceptives alone. The vector that reflects th is hypothesis is $x_{3}=(1,0,0,0,-1,0,0,0)$.

Including this vector into the design matrix in Table 5 yields a saturated model. Therefore, in order to have a model that can fail and that still allows us to test the hypothesis related to superadditivity we can either eliminate one of the vectors, $x_{1}$ or $x_{2}$, or place equality constraints. Typically, equality constraints posit the constraint that $\lambda_{1}=\lambda_{2}$ for $\mathrm{Q}=x_{1} \lambda_{1}+x_{2} \lambda_{2}$. If we pose this constraint the resulting new vector is $x_{4}=(2,1,1,0,-2,-1,-1,0)$. This vector, however, would imply some weighting. Therefore, we decide using some variant of setting parameters 
equal where there is no such weighting. We use the vectors, $x_{4}=(1,1,1,0$, $-1,-1,-1,0)$, and $x_{3}$ instead of $x_{1}$ and $x_{2}$, in Table 5 .

The costs and the benefits from placing this constraint are obvious. We gain the possibility to test the overall model and the superadditivity hypothesis. However, we forfeit the option to test the Independent Agents' Synergy and the Joint Agents' Synergy models against each other. They are no longer hierarchically related to each other. ${ }^{2}$

The Isolated Synergy Model. This proposes that, regardless of what the effects of single agents are, synergy is reflected by agents' interactions. Therefore, even if none of the single agents has any effect, there can still be synergy by way of agents' collaboration (interaction). The Isolated Synergy Model substitutes vector $x_{3}$ for vectors $x_{1}$ and $x_{2}$ in Table 5 .

\section{Fit of Synergy Models}

Table 6 displays the estimated expected cell frequencies for the four synergy models. Table 7 gives an overview of the overall model fit achieved by each of the four models.

The results in Tables 6 and 7 suggest the Bishop et al.'s (1975) nonhierarchical synergy model, the Independent Agents' and the Joint A gents' Synergy models provide excellent fit and are significantly better

TABLE 6

Observed and Estimated Expected Cell Frequencies for Four Synergy Models

\begin{tabular}{|c|c|c|c|c|c|}
\hline \multirow[b]{2}{*}{$T S U$} & \multirow{2}{*}{$\begin{array}{c}\text { Observed } \\
\text { Frequencies }\end{array}$} & \multicolumn{4}{|c|}{ Expected Cell Frequencies } \\
\hline & & BFH Model $1^{\mathrm{a}}$ & $I A S^{\mathrm{b}}$ & $J A S^{\mathrm{b}}$ & $I S^{\mathrm{b}}$ \\
\hline 111 & 14 & 14.00 & 12.34 & 14.00 & 14.00 \\
\hline 112 & 7 & 6.70 & 8.66 & 11.24 & 8.08 \\
\hline 121 & 12 & 12.00 & 13.66 & 7.76 & 5.75 \\
\hline 122 & 25 & 25.30 & 23.34 & 25.00 & 30.35 \\
\hline 211 & 2 & 2.10 & 3.66 & 2.00 & 2.00 \\
\hline 212 & 22 & 22.20 & 20.34 & 17.76 & 20.82 \\
\hline 221 & 8 & 7.91 & 6.34 & 12.24 & 14.43 \\
\hline 222 & 84 & 83.80 & 85.66 & 84.00 & 78.65 \\
\hline
\end{tabular}

${ }^{\mathrm{a}} \mathrm{BFH}$ is short for Bishop, Fienberg, and Holland (1975); results are from BFH, 1975, p. 113; ' $\mathrm{IAS}$, Independent Agents' Synergy Model; JA S, Joint Agents' Synergy Model; IS, Isolated Synergy Model.

\footnotetext{
${ }^{2}$ There is the option of placing the equality constraint in the Independent Agents' Synergy Model as well. However, this option would prevent us from estimating parameters for the two agents that can differ in magnitude and significance. Therefore, we will not apply this option in the Independent A gents' Model.
} 
TABLE 7

Goodness-of-Fit of Models in Tables 4 and 6

\begin{tabular}{lrccc}
\hline \multicolumn{1}{c}{ Model } & $L R-\chi^{2}$ & $d f$ & $\Delta L R-\chi^{2}$ & $\Delta d f$ \\
\hline Base [S, U][T] & 35.93 & 3 & - & - \\
BFH & 0.02 & 2 & 35.91 & 1 \\
IA S & 2.35 & 1 & 33.58 & 2 \\
JA S & 6.64 & 1 & 29.29 & 2 \\
IS & 12.74 & 2 & 23.19 & 1 \\
\hline
\end{tabular}

than the base model. The Isolated Synergy Model, although better than the base model, is not tenable itself. Therefore, it will not be considered further.

To come to a conclusion concerning the synergy hypotheses one can pursue two alternative routes. The first involves removing cell 111 as proposed by Bishop et al. (1975). The authors first conclude from their analyses that cell 111 is an outlier (for details see Bishop et al., 1975, p. 141; and Worcester, 1971). This result can also be obtained using configural frequency analysis (Lienert \& Krauth, 1975; von Eye, 1990). Second, Bishop et al. (1975, p. 114) conclude that their results support the hypothesis that the use of contraceptives is positively associated with thromboembolism, particularly among smokers. In contrast, among those who do not use oral contraceptives, smoking is not associated with the disease. The second route involves interpretation of those parameters that were specified to test the synergy model hypotheses. In the following paragraphs we pursue this route.

Fitting the Model of Independent Agents' Synergy. Using equation 4 and the transformation in equations 6 and 7, we now show that parameter interpretation can be highly problematic when design matrices are not orthogonal. Consider the matrix given in Table 5. The parameters for this matrix are of the form shown in Table 8.

TABLE 8

Parameters for Design Matrix in Table 5

\begin{tabular}{cl}
\hline Parameter & \multicolumn{1}{c}{ Expression } \\
\hline$\lambda_{0}$ & $1=8\left(\mu_{1}+\mu_{2}+\mu_{3}+\mu_{4}+\mu_{5}+\mu_{6}+\mu_{7}+\mu_{8}\right)$ \\
$\lambda_{T}$ & $1=8\left(\mu_{1}-\mu_{2}-\mu_{3}-3 \mu_{4}-\mu_{5}+\mu_{6}+\mu_{7}+3 \mu_{8}\right)$ \\
$\lambda_{S}$ & $1=8\left(-\mu_{1}-\mu_{2}+\mu_{3}+\mu_{4}-\mu_{5}-\mu_{6}+\mu_{7}+\mu_{8}\right)$ \\
$\lambda_{U}$ & $1=8\left(-\mu_{1}+\mu_{2}-\mu_{3}+\mu_{4}-\mu_{5}+\mu_{6}-\mu_{7}+\mu_{8}\right)$ \\
$\lambda_{x 1}$ & $1=4\left(\mu_{1}+\mu_{2}-\mu_{3}-\mu_{4}-\mu_{5}-\mu_{6}+\mu_{7}+\mu_{8}\right)$ \\
$\lambda_{x 2}$ & $1=4\left(\mu_{1}-\mu_{2}+\mu_{3}-\mu_{4}-\mu_{5}+\mu_{6}-\mu_{7}+\mu_{8}\right)$ \\
$\lambda_{S \times U}$ & $1=8\left(\mu_{1}-\mu_{2}-\mu_{3}+\mu_{4}+\mu_{5}-\mu_{6}-\mu_{7}+\mu_{8}\right)$ \\
\hline
\end{tabular}


Table 8 shows that application of the design matrix given in Table 5 leads to parameter estimates that are, with the exceptions of the constant and the main effect parameters for $S$ and $U$ and the interaction between $S$ and $U$, not reflective of the hypotheses expressed in form of contrasts. This applies in particular to the estimates of $\lambda_{x 1}$ and $\lambda_{x 2}$. In contrast, application of the transformation given in equations 6 and 7 leads to the parameter estimates given in Table 10. Table 9 displays the matrix $X^{*}$ which results from application of equation 6 .

Obviously, the design matrix in Table 9 does not look as if it would reflect the hypotheses specified in Table 5. However, the parameter estimates from $X^{*}$ do reflect these hypotheses, as is illustrated in Table 10. The parameter estimates for the design matrices, $\mathrm{X}$ and $\mathrm{X}^{*}$, appear in Table 11.

The overall model for both variants of $\mathrm{X}$ is the same: LR $\chi^{2}=2.35, d f=1, P=.125$. Thus, we conclude that the model describes the data adequately. However, a comparison of the parameter estimates suggests that the models are not equivalent. A lthough the $t$-values for some parameters are unchanged between $\mathrm{X}$ and $\mathrm{X}^{*}$, specifically, $\lambda_{0}, \lambda_{S}, \lambda_{U}$, and $\lambda_{S \times U}$, the $t$-values for others, specifically $\lambda_{T}, \lambda_{x 1}$, and $\lambda_{x 2}$, differ, one of them dramatically $\left(\lambda_{T}\right)$. As far as the model of

TABLE 9

Matrix $X^{*}$, the Result of Applying Equation 6 to the Design Matrix in Table 5

\begin{tabular}{crrrrrr}
\hline$c$ & $T$ & \multicolumn{1}{c}{$S$} & \multicolumn{1}{c}{$U$} & \multicolumn{1}{c}{$x_{1}$} & \multicolumn{1}{c}{$x_{2}$} & \multicolumn{1}{c}{$S \times U$} \\
\hline 0.125 & 0.125 & -0.125 & -0.125 & 0.25 & 0.25 & 0.125 \\
0.125 & -0.125 & -0.125 & 0.125 & 0.25 & -0.25 & -0.125 \\
0.125 & -0.125 & 0.125 & -0.125 & -0.25 & 0.25 & -0.125 \\
0.125 & -0.375 & 0.125 & 0.125 & -0.25 & -0.25 & 0.125 \\
0.125 & -0.125 & -0.125 & -0.125 & -0.25 & -0.25 & 0.125 \\
0.125 & 0.125 & -0.125 & 0.125 & -0.25 & 0.25 & -0.125 \\
0.125 & 0.125 & 0.125 & -0.125 & 0.25 & -0.25 & -0.125 \\
0.125 & 0.375 & 0.125 & 0.125 & 0.25 & 0.25 & 0.125 \\
\hline
\end{tabular}

TABLE 10

Param eters for Design Matrix in Table 5, After Transformation

\begin{tabular}{cl}
\hline Parameter & \multicolumn{1}{c}{ Expression } \\
\hline$\lambda_{0}$ & $\mu_{1}+\mu_{2}+\mu_{3}+\mu_{4}+\mu_{5}+\mu_{6}+\mu_{7}+\mu_{8}$ \\
$\lambda_{T}$ & $-\mu_{1}-\mu_{2}-\mu_{3}-\mu_{4}+\mu_{5}+\mu_{6}+\mu_{7}+\mu_{8}$ \\
$\lambda_{S}$ & $-\mu_{1}-\mu_{2}+\mu_{3}+\mu_{4}-\mu_{5}-\mu_{6}+\mu_{7}+\mu_{8}$ \\
$\lambda_{U}$ & $-\mu_{1}+\mu_{2}-\mu_{3}+\mu_{4}-\mu_{5}+\mu_{6}-\mu_{7}+\mu_{8}$ \\
$\lambda_{x 1}$ & $\mu_{1}+\mu_{2}-\mu_{5}-\mu_{6}$ \\
$\lambda_{x 2}$ & $\mu_{1}+\mu_{3}-\mu_{5}-\mu_{7}$ \\
$\lambda_{S \times U}$ & $\mu_{1}-\mu_{2}-\mu_{3}+\mu_{4}+\mu_{5}-\mu_{6}-\mu_{7}+\mu_{8}$ \\
\hline
\end{tabular}


TABLE 11

Parameter Estimates from the Design Matrices $X$ and $X^{*}$

\begin{tabular}{|c|c|c|c|c|c|c|c|}
\hline \multicolumn{4}{|c|}{ Parameter Estimates from $X$} & \multicolumn{4}{|c|}{ Parameter Estimates from $X^{*}$} \\
\hline & Value & $S E$ & $t$ & & Value & $S E$ & $t$ \\
\hline Intercept & 2.63 & 0.11 & 23.87 & Intercept & 21.04 & 0.88 & 23.87 \\
\hline$\lambda_{T}$ & 0.65 & 0.11 & 5.77 & $\lambda_{T}$ & 0.17 & 0.90 & 0.19 \\
\hline$\lambda_{S}$ & 0.38 & 0.10 & 3.88 & $\lambda_{S}$ & 3.08 & 0.79 & 3.88 \\
\hline$\lambda_{U}$ & 0.56 & 0.11 & 5.04 & $\lambda_{U}$ & 4.50 & 0.89 & 5.04 \\
\hline$\lambda_{x 1}$ & 0.22 & 0.20 & 1.12 & $\lambda_{x 1}$ & 0.36 & 0.69 & 0.52 \\
\hline$\lambda_{x 2}$ & 1.03 & 0.21 & 4.82 & $\lambda_{x 2}$ & 1.98 & 0.75 & 2.64 \\
\hline$\lambda_{S \times U}$ & 0.22 & 0.11 & 2.04 & $\lambda_{S \times U}$ & 1.78 & 0.87 & 2.04 \\
\hline
\end{tabular}

Independent Agents' Synergy is concerned, we conclude that the synergy hypothesis must be rejected despite the good overall model fit. One criterion for this model to be accepted was that two or more agents' effects must exist. The present results, however, suggest that only one agent's effect exists. This is the effect of U se of Oral Contraceptives. Smoking does not seem to have an effect above and beyond that of $U$. Thus, with only one effect present, the synergy hypothesis fails to be confirmed.

Fitting the Model of Joint Agents' Synergy. To fit the model of Joint A gents' Synergy we substitute the vectors, $x_{3}$ and $x_{4}$ for $x_{1}$ and $x_{2}$. The overall model fit is marginal: $\mathrm{LR}-\chi^{2}=6.46, d f=1$, and $P=.011$. The model can, therefore, be retained only if one sets $\alpha$ to 0.01 . Table 12 presents the parameter estimates for this model for both $\mathrm{X}$ and $\mathrm{X}$ *.

Even more so than Table 11, Table 12 suggests that results created without the transformation described in equations 6 and 7 can lead to inaccurate parameter estimates. Estimating parameters using $\mathrm{X}$ leads to the conclusion that both the combined vector of single agents' effects, $x_{4}$, and the vector that describes the superadditive component of these agents'

TABLE 12

Parameter Estimates from the Design Matrices $X$ and $X^{*}$

\begin{tabular}{|c|c|c|c|c|c|c|c|}
\hline \multicolumn{4}{|c|}{ Parameter Estimates from $X$} & \multicolumn{4}{|c|}{ Parameter Estimates from $X^{*}$} \\
\hline & Value & $S E$ & $t$ & & Value & $S E$ & $t$ \\
\hline Intercept & 2.60 & 0.12 & 21.07 & Intercept & 20.83 & 0.99 & 21.07 \\
\hline$\lambda_{T}$ & 0.61 & 0.11 & 5.32 & $\lambda_{T}$ & 0.18 & 0.98 & 0.18 \\
\hline$\lambda_{S}$ & 0.45 & 0.12 & 3.65 & $\lambda_{S}$ & 3.57 & 0.98 & 3.65 \\
\hline$\lambda_{U}$ & 0.63 & 0.12 & 5.16 & $\lambda_{U}$ & 5.06 & 0.98 & 5.16 \\
\hline$\lambda_{x 3}$ & 1.20 & 0.41 & 2.96 & $\lambda_{x 3}$ & 1.95 & 0.76 & 2.57 \\
\hline$\lambda_{x 4}$ & 0.38 & 0.19 & 2.04 & $\lambda_{x 4}$ & 1.03 & 0.96 & 1.08 \\
\hline$\lambda_{S \times U}$ & 0.14 & 0.12 & 1.14 & $\lambda_{S \times U}$ & 1.13 & 0.99 & 1.14 \\
\hline
\end{tabular}


synergy, $x_{3}$, capture significant portions of the variability in the table. However, after the proper transformation of the design matrix, it becomes clear that constraining the contrast of the agents to be equal yields nonsignificant parameters. Only the superadditivity parameter is significant. In the next section we ask whether the superadditivity contrast alone leads to a satisfactory data description.

Fitting the Model of Isolated Synergy. This requires to only use vector $x_{3}$ in the special hypotheses part of the model. Table 13 presents the parameter estimates for th is synergy model. Table 7 suggests that, although the Isolated Synergy Model is significantly better than the null model, it is not tenable by itself $\left(\mathrm{LR}-\chi^{2}=12.74, d f=2, P=.0017\right)$. Thus, parameters cannot be interpreted.

\section{Discussion of Synergy Models}

Overall, the investigation of synergy hypotheses via five models suggests that although some of the models provide excellent renderings of the data, none of them provides strong support of synergy hypotheses. The Bishop et al. (1975) model provides the best fit and supports the statement that the use of oral contraceptives "... is positively associated with thromboembolism, particularly among smokers ..." (p. 114). However, among those who do not smoke, smoking is not associated with thromboembolism. The Model of Independent Agents' Synergy suggests that while use of oral contraceptives leads to thromboembolism, smoking does not. The model of Joint Agents' Synergy provides marginal fit and suggests that there may be superadditivity in the sense that there is an effect that both agents have together beyond the single agents' effects. However, the single agents' effects had to be constrained to be equal for this model to become nonsaturated. The Isolated Synergy Model was not tenable. We thus conclude that the support of the synergy hypotheses for the present data is weak.

\section{TABLE 13}

Parameter Estimates from the Design Matrices $X$ and $X^{*}$

\begin{tabular}{|c|c|c|c|c|c|c|c|}
\hline \multicolumn{4}{|c|}{ Parameter Estimates from $X$} & \multicolumn{4}{|c|}{ Parameter Estimates from $X^{*}$} \\
\hline & Value & $S E$ & $t$ & & Value & $S E$ & $t$ \\
\hline Intercept & 2.48 & 0.13 & 18.54 & Intercept & 19.83 & 1.07 & 18.54 \\
\hline$\lambda_{T}$ & 0.49 & 0.11 & 4.37 & $\lambda_{T}$ & -0.06 & 0.86 & -0.07 \\
\hline$\lambda_{S}$ & 0.62 & 0.11 & 5.74 & $\lambda_{S}$ & 4.96 & 0.86 & 5.74 \\
\hline$\lambda_{U}$ & 0.77 & 0.11 & 7.10 & $\lambda_{U}$ & 6.19 & 0.87 & 7.10 \\
\hline$\lambda_{x 3}$ & 1.97 & 0.45 & 4.37 & $\lambda_{x 3}$ & 2.91 & 0.74 & 3.95 \\
\hline$\lambda_{S \times U}$ & -0.03 & 0.11 & -0.27 & $\lambda_{S \times U}$ & 3.70 & 0.86 & 4.30 \\
\hline
\end{tabular}




\section{SUMMARY AND OUTLOOK}

It was the goal of this paper to propose ways to model the concept of synergy. Three interpretations of the concept of synergy were considered. Using nonstandard log-linear models and a transformation recently proposed by Schuster (in preparation) these models were specified and tested. None of these models was specified in a way equivalent to hierarchical log-linear models or equivalent to models that can be created using elements from hierarchical models (this was done by Bishop et al., 1975).

Generalisations are conceivable in various ways. The following models can be specified using the methods presented in this article:

(1) models of partial synergy could be considered where synergy is identified to exist in subtables;

(2) models of synergy of developmental agents that act in a time-related way; for instance, agents can act either sequentially or synchronously (or both);

(3) group-specific synergy; for example, one can test the hypothesis that synergy occurs only in certain groups of individuals, but not in others; and

(4) longitudinal development of synergy; one can test assumptions concerning the time-related effectiveness of synergetic agents.

Many more models are conceivable, each of which can consider covariates. An additional group of methods is beyond the scope of this article, but not beyond the scope of what can be done. One can consider latent variable concepts of synergy and test these models using the methods proposed, for example, by Vermunt (1996).

Manuscript received September 1997

Revised manuscript received A pril 1998

\section{REFERENCES}

A lba, R.D. (1988). Interpreting the parameters of log-linear models. In J.S. Long (Ed.), Common problems/proper solutions (pp. 258-287). Newbury Park, CA: Sage.

A rnold, S.F., Klotz, D.M., Collins, B.M., Vonier, P.M., Guillette, L.J., \& McLachlan, J.A . (1996). Synergistic activation of estrogen receptor with combinations of environmental chemicals. Science, 272, 1489.

A ruin, A.S., A lmeida, G.L., \& Latasch, M.L. (1996). Organization of a simple two-joint synergy in individuals with Down syndrome. American Journal on Mental Retardation, $101,256-268$.

A yya, N., \& Lawless, H.T. (1992). Quantitative and qualitative evaluation of high-intensity sweeteners and sweetener mixtures. Chemical Senses, 17, 245-259. 
Bennett, C.M., Mlady, G.W., Fleshner, M., \& Rose, G.M. (1996). Synergy between chronic corticosterone and sodium azide treatments in producing a spatial learning deficit and inhibiting cytochrome oxidase activity. Proceedings of the National Academy of Sciences USA, 93, 1330.

Bishop, Y.M.M., Fienberg, S.E., \& Holland, P.W. (1975). Discrete multivariate analysis: theory and practice. Cambridge, M A: MIT Press.

Broglia, M., \& Brunori, A. (1994). Synergistic effect of low temperature and high sucrose concentration on maize pollen viability in aqueous medium. Crop Science, 34, 528.

Buckley, P.F., \& Schulz, S.C. (1996). Clozapine and risperidone: Refining and extending their use. Harvard Review of Psychiatry, 4, 184-199.

Clogg, C.C., Eliason, S.R., \& Grego, J.M. (1990). Models for the analysis of change in discrete variables. In A. von Eye (Ed.), Statistical methods in longitudinal research (Vol. II, pp. 409-441). Boston, M A : A cademic Press.

Drasner, K. (1988). Synergy between the antinociceptive effects of intrathecal clonidine and systemic morphine in the rat. Pain, 32, 309-312.

Elliott, G.C. (1988). Interpreting higher order interactions in log-linear analysis. Psychological Bulletin, 103, 121-130.

Gottlieb, G. (1992). Individual development and evolution. The genesis of novel behavior. New York: Oxford University Press.

Gunzburg, M. (1995). The use of combined individual, group, and marital therapy to resolve the narcissistic transference. International Journal of Group Psychotherapy, 45, 251-258.

Holt, T.D. (1979). Log-linear models for contingency table analysis: on the interpretation of parameters. Sociological Methods and Research, 11, 325-344.

Houston, D.A., \& Doan, K. (1996). Comparison of paired choice alternatives and choice conflict. Applied Cognitive Psychology, 10, 125-135.

Ketter, T.A. (1992). Synergy of carbamazepine and valproic acid in affective illness: Case report and review of the literature. Journal of Clinical Psychopharmacology, 12, 276-281.

Laffort, P., Etcheto, M., Patte, F., \& Marfaing, P. (1989). Implications of power law exponent in synergy and inhibition of olfactory mixtures. Chemical Senses, 14, 11-23.

Lienert, G.A., \& Krauth, J. (1975). Configural Frequency A nalysis as a statistical tool for defining types. Educational and Psychological Measurement, 35, 231-238.

Lodeon, J. (1986). Deux cas de non-consommation du marriage: Example de "strategie naturalistique." Genitif, 7, 40-41.

Long, J.S. (1984). Estimable functions in log-linear models. Sociological Methods and Research, 12, 399-432.

McCullagh, P., \& Nelder, J.A . (1983). Generalized linear models. London: Chapman \& Hall. Miaskowski, C. (1993). A ntinociception produced by receptor selective opioids: Modulation of supraspinal antinociceptive effects by spinal opioids. Brain Research, 608, 87-94.

Nelson, T.O. (1996). Consciousness and metacognition. American Psychologist, 51, 102-116. R indskopf, D . (1990). Nonstandard log-linear models. Psychological Bulletin, 108, 150-162. Seale, T.W., Carney, J.M., Rennert, O.M., \& Flux, M. (1987). Coincidence of seizure susceptibility to caffe ine and to the benzodiazepine inverse agonist, DMCM, in SWR and CBA inbred mice. Pharmacology, Biochemistry and Behavior, 26, 381-387.

Schuster, C. (in prep). A simplified procedure for testing hypotheses in hierarchical and nonstandard log-linear models.

Sloane, D., \& Morgan, S.P. (1996). An introduction to categorical data analysis. Annual Review of Sociology, 22, 351-375.

Sobe1, M.E. (1995). Causal inference in the social and behavioral sciences. In G. Arminger, C.C. Clogg, \& M.E. Sobel (Eds.), Handbook of statistical modeling for the social and behavioral sciences (pp. 1-38). New York: Plenum. 
Vermunt, J.K. (1996). Log-linear models for event history analysis. Thousand Oaks, CA: Sage.

von Eye, A. (1990). Introduction to Configural Frequency Analysis: The search for types and antitypes in cross-classifications. Cambridge, UK: Cambridge University Press.

von Eye, A., \& Brandtstädter, J. (in press). The Wedge, the Fork, and the Chain-Modeling concepts of developmental dependency using manifest categorical variables. Psychological Methods.

von Eye, A., Kreppner, K., \& Weßels, H. (1994). Log-linear modeling of categorical data in developmental research. In D.L. Featherman, R.M. Lerner, \& M. Perlmutter (Eds.), Lifespan development and behavior (pp. 225-248). Hillsdale, $\mathrm{NJ}$ : Erlbaum.

von Eye, A., \& Spie1, C. (1996). Standard and non-standard log-linear symmetry models for measuring change in categorical variables. The American Statistician, 50, 300-305.

Wilens, T.E., Spencer, T., Biederman, J., \& Wozniak, J. (1995). Combined pharmacotherapy: An emerging trend in pediatric psychopharmacology. Journal of the American Academy of Child and Adolescent Psychiatry, 34, 110-112.

Worcester, J. (1971). The relative odds in the $2^{3}$ contingency table. American Journal of Epidemiology, 93, 145-159. 